\title{
Symmetry adapted analysis of the magnetic and structural phase diagram of $\mathrm{Bi}_{1-x} \mathrm{Y}_{x} \mathrm{CrO}_{3}$
}

\author{
Claire V. Colin, ${ }^{*}$ Alejandro Gómez Pérez, Pierre Bordet, Céline Goujon, and Céline Darie \\ Institut Néel, CNRS et Université Joseph Fourier, B.P. 166, F-38042 Grenoble Cédex 9, France
}

(Received 17 February 2012; published 4 June 2012)

\begin{abstract}
The effect of the substitution of yttrium for bismuth in $\mathrm{BiCrO}_{3}$ distorted perovskite has been investigated by means of magnetic measurements and symmetry mode analysis of neutron and x-ray powder diffraction data. $\mathrm{Bi}_{1-x} \mathrm{Y}_{x} \mathrm{CrO}_{3}$ compounds were synthesized under high pressure and high temperature. Magnetic and structural phase diagrams were established by means of magnetization measurements and x-ray and neutron powder diffraction. We found that the monoclinic distorted structure is replaced by an orthoferrite-type orthorhombic structure at $x \geqslant 0.05$. Symmetry adapted analysis was performed, indicating the importance of the LD3 distortion mode for the stabilization of the monoclinic structure and its antiferroelectric arrangement. The interplay between crystal distortion modes and magnetic properties is discussed.
\end{abstract}

DOI: 10.1103/PhysRevB.85.224103

PACS number(s): 61.50.Ah, 75.25.-j, 75.85.+t, 77.80.-e

\section{INTRODUCTION}

Bismuth-based perovskite compounds $\mathrm{BiMO}_{3} \quad(\mathrm{M}=$ transitionmetal) have recently attracted a lot of attention due to the possibility of intrinsic multiferroism. Mutual control of polarization and magnetization by applying a magnetic or electric field in such materials could have considerable potential applications. One member of the family, bismuth ferrite $\mathrm{BiFeO}_{3}$, is a rare single-phase multiferroic, with ferroelectric and antiferromagnetic order at room temperature. In this compound, magnetic properties are due to the $d$ electrons of $\mathrm{Fe}^{3+}$ cations, whereas ferroelectricity originates from the stereochemical activity of the $\mathrm{Bi}^{3+}$ cation electronic lone pair, which favors a noncentrosymmetric structural distortion. One of the least investigated compounds in the series is $\mathrm{BiCrO}_{3}$, probably because it can only be synthesized under high pressure. Bismuth chromite was first reported by Sugawara et al., ${ }^{1,2}$ with a triclinic structure and weak ferromagnetic properties. During the last decade, renewed interest has developed following theoretical predictions by Hill et al., ${ }^{3}$ who proposed a G-type antiferromagnetic ground state, with an antiferrodistortive or antiferroelectric structural distortion. The crystal structure of $\mathrm{BiCrO}_{3}$ was debated for a long time. It was first reported as a strongly distorted perovskite with monoclinic polar symmetry (space group $C 2){ }^{4}$ A structural phase transition from a monoclinic structure to an orthorhombic one (orthoferrite type with space group Pnma) occurs at about $420 \mathrm{~K}$, accompanied by an anomaly of the dielectric permittivity. A rise in magnetic susceptibility is observed at $114 \mathrm{~K}$ and a difference between zero-fieldcooled (ZFC) and field-cooled (FC) magnetization curves appears below $75 \mathrm{~K}$, with an opening of the hysteresis loop. More recently, Belik et al. ${ }^{5}$ reinvestigated the crystal and magnetic structures of $\mathrm{BiCrO}_{3}$ using neutron powder diffraction. The structure of the orthorhombic phase above $420 \mathrm{~K}$ was confirmed. On the other hand, those authors proposed a centrosymmetric symmetry with space group $C 2 / c$ for the low-temperature monoclinic phase, which is then incompatible with ferroelectricity. Neutron diffraction data revealed the establishment of antiferromagnetic long-range order below the magnetic transition temperature $T_{N}=109 \mathrm{~K}$. An antiferromagnetic G-type structure was refined with spins lying along the $b$ axis of the monoclinic cell. Darie et al. ${ }^{6}$ confirm by neutron powder diffraction the room-temperature centrosymmetric space group $C 2 / c$ and showed the occurrence of a spin reorientation process below Néel temperature at $T_{\mathrm{SR}}$ around $80 \mathrm{~K}$, coinciding with the $\mathrm{ZFC}-\mathrm{FC}$ separation in the magnetization curves. The magnetism of $\mathrm{BiCrO}_{3}$ can be qualitatively understood in terms of the superexchange mechanism via $C r_{1}\left(t_{2 g}\right)-\mathrm{O} 2 p-C r_{2}\left(t_{2 g}\right){ }^{7}$

Understanding multiferroism in a complex material such as $\mathrm{BiCrO}_{3}$ cannot be envisaged without establishing the close relationship between structural and magnetic properties. To do so we propose here the following approach: (i) the structural distortion of $\mathrm{BiCrO}_{3}$ will be tuned by isovalent substitution of the $\mathrm{Bi}^{3+}$ cation by another cation which does not possess a stereoactive electronic lone pair; and (ii) the magnetic structures and active distortion modes will be determined by refinement of neutron and $\mathrm{x}$-ray powder diffraction data using the symmetry mode analysis concept. This method allows the hierarchization of distortion modes and identification of those which stabilize the various distorted structures with respect to the cubic perovskite one. Since the structural distortions are related to the magnetic superexchange through the control of orbital overlaps, this procedure is well suited to reveal the links between structure and magnetic ordering in this quite complex system.

We chose to study the lone-pair dilution effect on the crystal and magnetic structures of $\mathrm{BiCrO}_{3}$ by substituting $\mathrm{Y}^{3+}$ for $\mathrm{Bi}^{3+}$. Indeed both cations are isovalent, have compatible ionic radii, and are nonmagnetic. At variance with $\mathrm{Bi}^{3+}$, the $\mathrm{Y}^{3+}$ cation does not possess a stereochemical active electronic lone pair. $\mathrm{YCrO}_{3}$ yttrium chromite crystallizes with an orthoferrite-type structure, with Pnma centrosymmetric space-group symmetry. It behaves as a weak ferromagnet, with a spontaneous moment appearing only along the $b$ axis. $^{8}$ It presents a G-type magnetic arrangement below $\mathrm{T}_{N}=140 \mathrm{~K}$ as established by Bertaut using neutron powder diffraction. ${ }^{9}$ Recently, $\mathrm{YCrO}_{3}$ has been considered a potential multiferroic compound. The possible existence of noncentrosymmetry at the local scale ${ }^{10}$ was reported to account for a peak observed in the permittivity curve, the small value of the polarization in thin films, ${ }^{11}$ and an anomaly in bulk dielectric properties. ${ }^{12}$ We present below the sample preparation, magnetic measurements, and neutron powder 
diffraction study of the complete solid solution $\mathrm{Bi}_{1-x} \mathrm{Y}_{x} \mathrm{CrO}_{3}$ for $0 \leqslant x \leqslant 1$.

\section{METHODS}

$\mathrm{Bi}_{1-x} \mathrm{Y}_{x} \mathrm{CrO}_{3}$ polycrystalline samples with compositions of $x=0.01,0.05,0.2$, and 0.5 were synthesized by solidstate reaction under HPHT in a Conac anvil-type apparatus. Stoichiometric amounts of high-purity $\mathrm{Bi}_{2} \mathrm{O}_{3}, \mathrm{Y}_{2} \mathrm{O}_{3}$, and $\mathrm{Cr}_{2} \mathrm{O}_{3}$ were intimately mixed, placed in $\mathrm{Au}$ capsules, and treated at $4 \mathrm{GPa}$ and $1213 \mathrm{~K}$ for $30 \mathrm{~min}$. The use of $d=10 \mathrm{~mm}$ (Conac 40) capsules allowed us to synthesize $1 \mathrm{~g}$ at a time. ${ }^{6,13}$ For the synthesis of $\mathrm{YCrO}_{3}$ the combustion method mentioned by Bedekar et al. ${ }^{14}$ was used. Yttrium nitrate $\left[\mathrm{Y}\left(\mathrm{NO}_{3}\right) 36 \mathrm{H}_{2} \mathrm{O} ; 99.9 \%\right.$ pure] and chromium nitrate $\left[\mathrm{Cr}\left(\mathrm{NO}_{3}\right) 39 \mathrm{H}_{2} \mathrm{O} ; 99.9 \%\right.$ pure] were used as oxidants. Glycine $\left(\mathrm{NH}_{2} \mathrm{CH}_{2} \mathrm{COOH}\right)$ was used as fuel. The conditions were a fuel excess with an oxidant:fuel ratio equal to 1:2. An autoignition reaction was initiated by heating the mixture at ca. $420 \mathrm{~K}$, after which a 6-h treatment was performed in a furnace at 1273 K. Energy Dispersive X-Ray spectroscopy confirmed the expected stoichiometry for all samples.

After synthesis, the phase purity and crystal structure were characterized by powder $\mathrm{x}$-ray diffraction at room temperature on a Bruker D8 diffractometer $(\mathrm{CuK} \alpha$ radiation; $\lambda=$ $1.5406 \AA$ ). The magnetic structures of selected samples were investigated by neutron powder diffraction using the two-axis diffractometers D1B at Institut Laue Langevin (Grenoble) and G4-1 at Laboratoire Léon Brillouin (Saclay). The wavelengths used for the measurements were 2.52 and $2.43 \AA$, respectively, corresponding to the (002) Bragg reflection of a pyrolitic graphite monochromator. Due to these high wavelengths and good angular resolution at low diffraction angles, both instruments are well adapted to magnetic structure investigations. For each experiment, approximately $1 \mathrm{~g}$ of sample was placed in a vanadium can inside an "orange" LHe cryostat. Some of the samples were made by mixing the products of two synthesis experiments. In such cases, these products were checked to display the same magnetic transition temperatures. All diffraction data analyses were done using the FullProf Suite software package. ${ }^{15}$ Magnetization measurements as a function of temperature and magnetic field were carried out in a Quantum Design superconducting quantum interference device. The free powder was wrapped in plastic film and put in a straw. Measurements were carried out using a FC-ZFC procedure under a magnetic field of 1000 Oe. Hysteresis loops were recorded at magnetic ordering temperatures of $2 \mathrm{~K}$ and above to check for the presence of magnetic impurities. Magnetic transition temperatures (Néel temperature and spin reorientation temperature) were determined by derivation of the magnetization curves.

\section{RESULTS}

\section{A. X-ray powder diffraction}

Powder X-ray diffraction patterns obtained for the synthesized samples are shown in Fig. 1. All samples were found close to single phase. The main impurities detected were bismuth oxycarbonate $\mathrm{Bi}_{2} \mathrm{O}_{2} \mathrm{CO}_{3}$ (bismutite), a phase which may be identified as a cubic bismuth oxide with body-centered



FIG. 1. (Color online) X-ray diffraction pattern of $\mathrm{Bi}_{1-x} \mathrm{Y}_{x} \mathrm{CrO}_{3}$ compounds. Impurity phases: bismuthite $\mathrm{Bi}_{2} \mathrm{O}_{2} \mathrm{CO}_{3}$ (asterisks), cubic bismuth oxide $\gamma-\mathrm{Bi}_{2} \mathrm{O}_{3}$ (cross), and chromium oxide $\mathrm{Cr}_{2} \mathrm{O}_{3}$ (open circle).

symmetry $\gamma-\mathrm{Bi}_{2} \mathrm{O}_{3}$, but which could also incorporate chromium cations and chromium oxide $\mathrm{Cr}_{2} \mathrm{O}_{3}$. Figure 1 clearly evidences a change in symmetry for $0.01 \leqslant x \leqslant 0.05$. For $x \leqslant 0.01$, the patterns were indexed with the $C 2 / c$ monoclinic space group (No. 15), and for $x \geqslant 0.05$, orthorhombic symmetry with the Pnma space group (No. 62 ). Figure 2 illustrates the refined cell parameters as a function of the yttrium concentration, $x$, expressed using a pseudocubic perovskite cell. The $a_{\mathrm{pc}}, b_{\mathrm{pc}}$, and $c_{\mathrm{pc}}$ cell parameters decrease upon $\mathrm{Y}$ substitution, which is expected considering the difference in ionic radius between $\mathrm{Bi}^{3+}(117 \mathrm{pm})$ and $\mathrm{Y}^{3+}(104$ pm) cations. Within the solid-solution domain with Pnma symmetry $(x \geqslant 0.05)$, the cell parameters follow Vegard's law, with a linear evolution against cationic composition (Fig. 2). On the other hand, a jump is observed in the lattice parameters

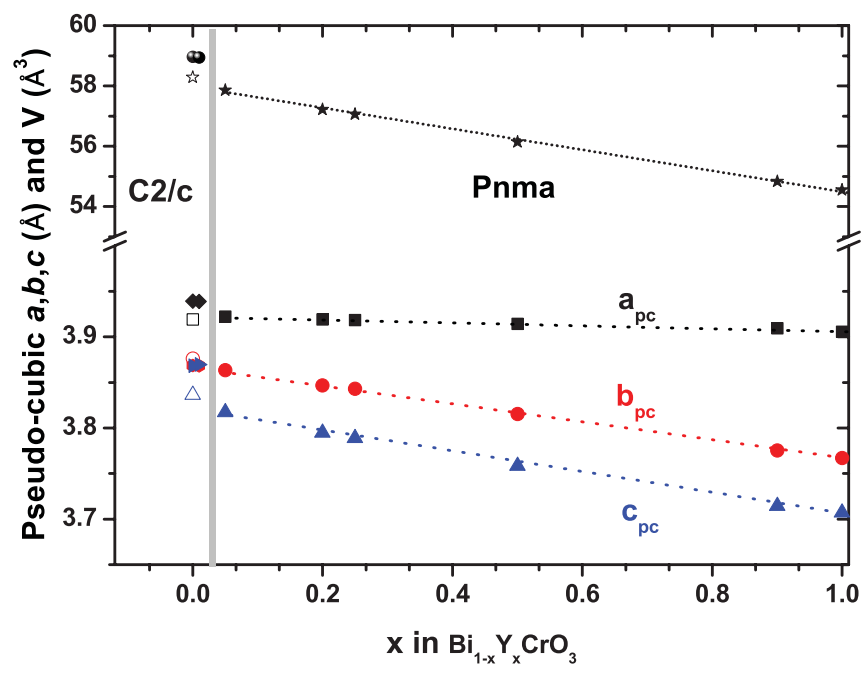

FIG. 2. (Color online) Evolution of room-temperature pseudocubic lattice parameters and volume for $\mathrm{Bi}_{1-x} \mathrm{Y}_{x} \mathrm{CrO}_{3}$ compounds (filled symbols). Parameters for $\mathrm{BiCrO}_{3} x=0$ taken from Darie et al., ${ }^{6}$ determined at $430 \mathrm{~K}$ for the high-temperature Pnma phase (openy symbols). Uncertainties are less than the size of the symbols. 
TABLE I. Structural and magnetic properties of $\mathrm{Bi}_{1-x} \mathrm{Y}_{x} \mathrm{CrO}_{3}$ compounds.

\begin{tabular}{lcccccc}
\hline \hline Compound & Space group & $\begin{array}{c}\text { Lattice parameters } \\
(\AA)\end{array}$ & $\begin{array}{c}T_{N} \\
(\mathrm{~K})\end{array}$ & $\begin{array}{c}T_{\mathrm{SR}} \\
(\mathrm{K})\end{array}$ & $\begin{array}{c}\text { Saturation magnetization } \\
\left(\mu_{B} / \text { f.u. }\right)\end{array}$ & $\begin{array}{c}\text { Estimated canting angle } \\
(\mathrm{deg})\end{array}$ \\
\hline $\mathrm{BiCrO}_{3}$ & $C 2 / c$ & $9.4681(2), 5.4822(1), 9.5865(2), 108.576(2)$ & 109 & 75 & & \\
$\mathrm{Bi}_{0.99} \mathrm{Y}_{0.01} \mathrm{CrO}_{3}$ & $C 2 / c$ & $9.4655(2), 5.4814(1), 9.5883(2), 108.584(2)$ & 112 & 74 & $0.026(1)$ & $0.50(6)$ \\
$\mathrm{Bi}_{0.95} \mathrm{Y}_{0.05} \mathrm{CrO}_{3}$ & Pnma & $5.5470(2), 7.7272(1), 5.3986(3)$ & 160 & & $0.015(1)$ & $0.29(6)$ \\
$\mathrm{Bi}_{0.80} \mathrm{Y}_{0.20} \mathrm{CrO}_{3}$ & Pnma & $5.5427(2), 7.6933(3), 5.3670(2)$ & 151 & $0.017(1)$ & $0.32(6)$ \\
$\mathrm{Bi}_{0.50} \mathrm{Y}_{0.50} \mathrm{CrO}_{3}$ & Pnma & $5.5355(8), 7.6308(3), 5.3154(8)$ & 141 & $0.024(1)$ & $0.45(6)$ \\
$\mathrm{YCrO}_{3}$ & Pnma & $5.5237(1), 7.5343(2), 5.2427(1)$ & 140 & & $0.020(1)$ & $0.38(6)$ \\
\hline \hline
\end{tabular}

and volume evolution between $x=0.01$ and $x=0.05$, correlated with the change in symmetry from $C 2 / c$ monoclinic to Pnma orthorhombic. It is worth noting that the Pnma structure is the one adopted by $\mathrm{YCrO}_{3}$ at room temperature but also by $\mathrm{BiCrO}_{3}$ at high temperature (above $T=420 \mathrm{~K}$ ). ${ }^{13}$ Cell parameters of the $\mathrm{BiCrO}_{3}$ phase with Pnma symmetry obtained at $430 \mathrm{~K}^{6}$ (open symbols) are reported in Fig. 2 to illustrate the continuity of their evolution over the whole phase diagram. Structural data are summarized in Table I.

\section{B. Magnetization measurements}

Figure 3 shows two typical magnetization curves for $\mathrm{Bi}_{1-x} \mathrm{Y}_{x} \mathrm{CrO}_{3}$ samples. Samples with monoclinic symmetry (Bi-rich side of the phase diagram) behave similarly to $\mathrm{BiCrO}_{3}$ : the Néel temperature is observed at about $112 \mathrm{~K}$, while at $T_{\mathrm{SR}}=73 \mathrm{~K}$, a spin reorientation transition occurs where FC and ZFC curves separate. This is the signature for the appearance of weak ferromagnetism. For samples crystallizing with orthorhombic symmetry $(x \geqslant 0.05)$, the behavior is similar to that of $\mathrm{YCrO}_{3}$, with $\mathrm{FC}$ and $\mathrm{ZFC}$ curves separating below the Néel transition and no sign of spin reorientation. The typical magnetic field dependence of the magnetization is displayed in the inset in Fig. 3. All samples show an hysteresis loop at low temperature, however, the saturation magnetization observed is about 100 times smaller than that expected for an $S=3 / 2$ ferromagnet, i.e., $\approx 3 \mu_{B}$. Such a weak saturation magnetization is probably the result of a small non



FIG. 3. (Color online) Field-cooled (filled symbols) and zerofield-cooled (open symbols) magnetization curves recorded under a magnetic field of 1000 Oe for $\mathrm{Bi}_{0.99} \mathrm{Y}_{0.01} \mathrm{CrO}_{3}$ and $\mathrm{Bi}_{0.95} \mathrm{Y}_{0.05} \mathrm{CrO}_{3}$. Inset: Magnetic field dependence of the magnetization for $\mathrm{Bi}_{0.95} \mathrm{Y}_{0.05} \mathrm{CrO}_{3}$ at 2, 180, and $300 \mathrm{~K}$. compensated canting of the spins. Canting angle $\alpha$ can be estimated using a simple relation between saturation magnetization representing the uncompensated moment (ferromagnetic component; $M_{S}$ ) and that representing the orbital quenched $\mathrm{Cr}^{3+}$ moment:

$$
\tan \alpha=\frac{M_{S}}{g_{J} \mu_{B} S} .
$$

Very small canting angles (lower than $1^{\circ}$ ) are estimated. Table I summarizes magnetization data for all samples.

\section{Determination of the magnetic structure}

Neutron diffraction measurements were carried out on powders of $\mathrm{Bi}_{1-x} \mathrm{Y}_{x} \mathrm{CrO}_{3}$ for $x=0.01,0.05,0.2,0.5$, and 1 over a wide temperature range. Figure 4 shows typical evolutions of diffractograms versus temperature. For each sample, magnetic diffraction peaks clearly appear at low temperatures. It was easily found that all magnetic contributions can be indexed with the nuclear unit cell, i.e., with a magnetic propagation vector $\mathbf{k}=0$.

The magnetic structure determination of all samples was done by symmetry analysis, following the representation analysis technique described by Bertaut. ${ }^{16}$ Calculations were carried out using version $2 \mathrm{~K}$ of the program SARA $h$-Representational Analysis ${ }^{17}$ and BasIreps integrated in FullProf Suite. ${ }^{18}$

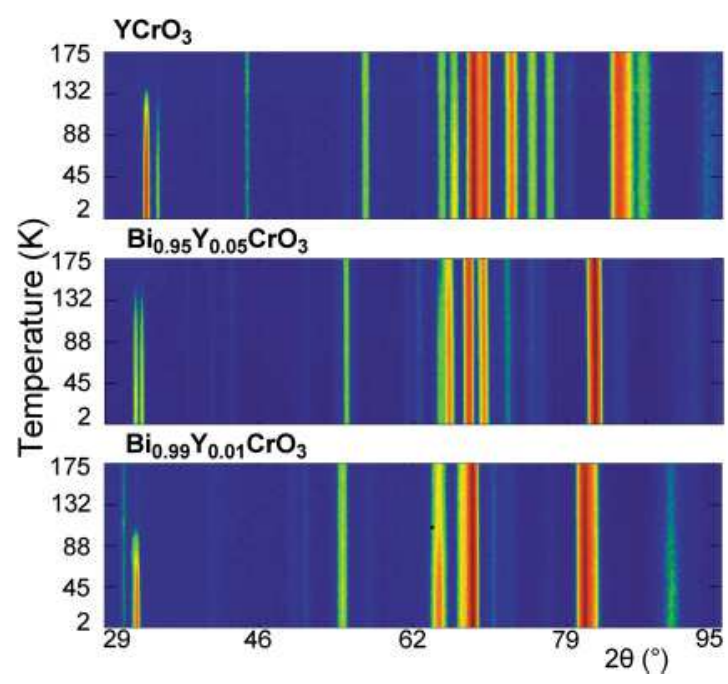

FIG. 4. (Color online) Thermal evolution of neutron powder diffraction patterns of $\mathrm{YCrO}_{3}, \mathrm{Bi}_{0.95} \mathrm{Y}_{0.05} \mathrm{CrO}_{3}$, and $\mathrm{Bi}_{0.99} \mathrm{Y}_{0.01} \mathrm{CrO}_{3}$ compounds. 
TABLE II. Basis vectors for space group Pnma with $\mathbf{k}_{19}=$ $(0,0,0)$. The decomposition of the magnetic representation for the $\mathrm{Cr}$ site $(0.5,0.5,0)$ is $\Gamma_{\mathrm{Mag}}=3 \Gamma_{1}^{1}+0 \Gamma_{2}^{1}+3 \Gamma_{3}^{1}+0 \Gamma_{4}^{1}+3 \Gamma_{5}^{1}+0 \Gamma_{6}^{1}+$ $3 \Gamma_{7}^{1}+0 \Gamma_{8}^{1}$. Atoms of the nonprimitive basis are defined as follows: $1,(0.5,0.5,0) ; 2,(0,0,0.5) ; 3,(0.5,0,0)$; and $4,(0,0.5,0.5)$. For notation see Wollan et al. ${ }^{19}$

\begin{tabular}{lcccc}
\hline \hline IR & $m_{\| a}$ & $m_{\| b}$ & $m_{\| c}$ & Shubnikov group \\
\hline$\Gamma_{1}$ & $\mathrm{G}$ & $\mathrm{C}$ & $\mathrm{A}$ & $P n m a$ \\
$\Gamma_{3}$ & $\mathrm{~B}$ & $\mathrm{~A}$ & $\mathrm{C}$ & $P n m^{\prime} a^{\prime}$ \\
$\Gamma_{5}$ & $\mathrm{~A}$ & $\mathrm{~B}$ & $\mathrm{G}$ & $P n^{\prime} m a^{\prime}$ \\
$\Gamma_{7}$ & $\mathrm{C}$ & $\mathrm{G}$ & $\mathrm{B}$ & $P n^{\prime} m^{\prime} a$ \\
\hline \hline
\end{tabular}

\section{Samples with orthorhombic symmetry}

Let us first examine the magnetic structures of samples with $x \geqslant 0.05$ for which the nuclear structure has Pnma symmetry. For the propagation vector $\mathbf{k}=\mathbf{0}$, the small group $G_{k}$, formed by those elements of space group Pnma which leave $\mathbf{k}$ invariant, coincides with the space group Pnma itself. For $\mathbf{k}=\mathbf{0}$, the group $G_{k}$ is composed of eight irreducible representations (IRs). A representation $\Gamma$ is constructed with the Fourier components $m^{k}$ corresponding to the four $\mathrm{Cr}$ atoms located at Wyckoff position $4 b$, denoted (1) $1,(1 / 2,1 / 2,0) ; 2,(0,0,1 / 2)$; $3,(1 / 2,0,0)$; and $4,(0,1 / 2,1 / 2)$. The decomposition of the magnetic representation for the $\mathrm{Cr}$ site is $\Gamma_{\mathrm{Mag}}=3 \Gamma_{1}^{1}+0 \Gamma_{2}^{1}+$ $3 \Gamma_{3}^{1}+0 \Gamma_{4}^{1}+3 \Gamma_{5}^{1}+0 \Gamma_{6}^{1}+3 \Gamma_{7}^{1}+0 \Gamma_{8}^{1}$. The presence of four $\mathrm{Cr}^{3+}$ cations in the unit cell leads to a large variety of different couplings between them and thus to many possible magnetic structures, which may be antiferromagnetic or ferromagnetic. To describe the spin configurations, we use the notation of Wollan et al. ${ }^{19}$ for spins in a pseudocubic lattice commonly used for perovskite structures. The different basis vectors associated with each IR and calculated by using the projection operator technique are presented in Table II. The labeling of the propagation vector and the IRs follows the scheme used by Kovalev. ${ }^{20}$

All possible models deduced from the symmetry analysis were tested by Rietveld refinement. For compositions larger than $x \geqslant 0.5$, a satisfactory fit to the data could only be obtained for the model described by the IR $\Gamma_{5}$ (see Fig. 5). Within experimental resolution, we could not find a magnetic moment component along the $a$ or $b$ direction of the orthorhombic unit cell, but only along $c$. The observed, calculated, and difference patterns for the final refinement are
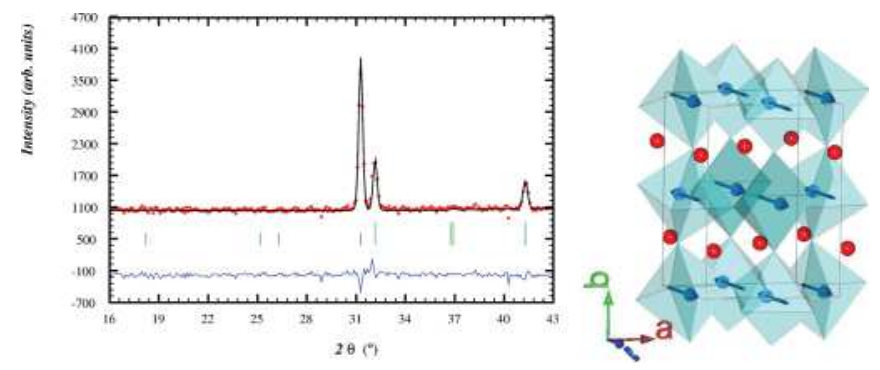

FIG. 5. (Color online) Left: Fragment of the low-angle range of the G4.1 neutron diffraction pattern of $\mathrm{Bi}_{0.5} \mathrm{Y}_{0.5} \mathrm{CrO}_{3}$ at $1.7 \mathrm{~K}$ refined with the $\Gamma_{5}$ model. Right: Refined G-type magnetic structure consisting of $\mathrm{Cr}^{3+}$ spins lying along the $c$ axis, $P n^{\prime} m a^{\prime}$ symmetry.

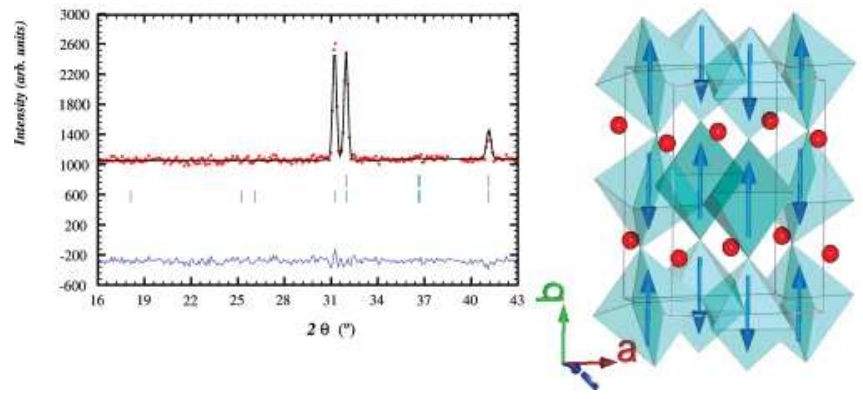

FIG. 6. (Color online) Left: Fragment of the low-angle range of the D1b neutron diffraction pattern of $\mathrm{Bi}_{0.8} \mathrm{Y}_{0.2} \mathrm{CrO}_{3}$ at $1.7 \mathrm{~K}$ refined with the $\Gamma_{7}$ model. Right: Refined G-type magnetic structure consisting of $\mathrm{Cr}^{3+}$ spins lying along the $b$ axis, $P n^{\prime} m^{\prime} a$ symmetry.

shown in Fig. 5. A scheme of the magnetic structure is given in the inset. The magnetic arrangement is that of a G-type antiferromagnet with all $\mathrm{Cr}^{3+}$ cations coupled antiparallel to their six nearest neighbors (notation of Ref. 19), all spins being aligned along the $c$ direction. This magnetic structure is the same as the one reported by Bertaut ${ }^{9}$ for pure $\mathrm{YCrO}_{3}$. As shown above, magnetic measurements indicate the presence of weak ferromagnetism, and therefore some canting of the spins should be observed. However, we were not able to detect any ferromagnetic component with the NPD data. This can be understood given the weakness of this ferromagnetic component seen by magnetization measurements $\left(\approx 0.02 \mu_{B}\right)$, which is probably below the detection limit for neutron powder diffraction. Nevertheless, it is noteworthy that such canting would be allowed by symmetry for the B-type ferromagnetic arrangement with spins components aligned along the $b$ axis. The values of the refined magnetic moments of $\mathrm{Cr}^{3+}$ are plotted vs temperature in Fig. 7. At $2 \mathrm{~K}$, the moment reaches a value close to $2.5 \mu_{B}$. This magnetic moment is comparable to the saturation value for the spin-only free-ion form factor for $\mathrm{Cr}^{3+}$ determined experimentally by Brown et al. ${ }^{21}$

For samples with $x \leqslant 0.2$, the best fit to the data was obtained for the $\Gamma_{7}$ representation (see Fig. 6). Within experimental resolution, no spin components were found along the $a$ and $c$ directions. The magnetic structure corresponds again to a G-type structure, but in this case the spins are oriented along the $b$ direction. A ferromagnetic component along the $c$ axis is allowed by symmetry, but none could be detected in our analysis. Refinement of the $\mathrm{Cr}^{3+}$ magnetic moment yields values at $2 \mathrm{~K}$ similar to those for samples with the $\Gamma_{5}$ magnetic structure (see Fig. 7).

\section{Samples with monoclinic symmetry}

We now consider the determination of magnetic structure of the sample with monoclinic symmetry, $x \leqslant 0.01$. Symmetryallowed magnetic structures were again determined by representational analysis. The crystal structure of $\mathrm{Bi}_{0.99} \mathrm{Y}_{0.01} \mathrm{CrO}_{3}$ above the magnetic ordering temperature was supposed to be the $C 12 / c 1$ space group (No. 15:b1). This hypothesis follows the proposition of previous authors and is confirmed in the next section. The $C 2 / c$ space group involves two centering operations and four symmetry operations. Of these symmetry operations, four leave the propagation $\mathbf{k}$ invariant or transform it into an equivalent vector. The decomposition of the magnetic 

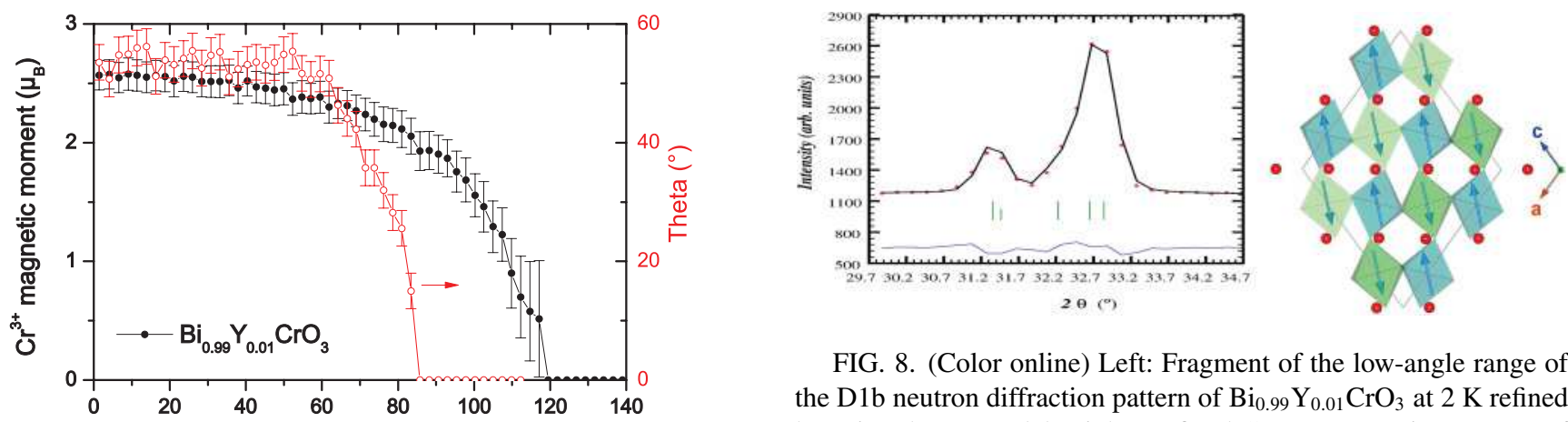

FIG. 8. (Color online) Left: Fragment of the low-angle range of the D1b neutron diffraction pattern of $\mathrm{Bi}_{0.99} \mathrm{Y}_{0.01} \mathrm{CrO}_{3}$ at $2 \mathrm{~K}$ refined by using the $\Gamma_{3}$ model. Right: Refined G-type magnetic structure of $\mathrm{Cr}^{3+}$ spins lying in the $(a, c)$ plane, $C 2^{\prime} / c^{\prime}$ symmetry.

(1/4,1/4,1/2); $C r 1_{2},(3 / 4,1 / 4,0) ; C r 2_{1},(0,0.235,3 / 4)$; and $\mathrm{Cr} 2_{2},(0,0.765,1 / 4)$. For each site, there are two possibilities for spin coupling:

$$
\begin{gathered}
M=S 1_{1}+S 1_{2}, \\
L=S 1_{1}-S 1_{2} .
\end{gathered}
$$

Combining the two sites leads to several possible antiferromagnetic structures. To simplify the problem we constrain the magnetic moments to have the same magnitude and consider only collinear structures. Then four possible arrangements remain:

$$
\begin{aligned}
& F=S 1_{1}+S 2_{1}+S 1_{2}+S 2_{2}, \\
& C=S 1_{1}+S 2_{1}-S 1_{2}-S 2_{2}, \\
& G=S 1_{1}-S 2_{1}+S 1_{2}-S 2_{2}, \\
& A=S 1_{1}-S 2_{1}-S 1_{2}+S 2_{2} .
\end{aligned}
$$

These possibilities were checked by Rietveld refinement. The best fit was obtained for the $\Gamma_{3}$ magnetic representation (see Fig. 8). The structure refined at $2 \mathrm{~K}$ is a G-type structure with spins aligned within the $(a, c)$ plane of the monoclinic unit cell, forming an angle $\Theta$ of 55(2) ${ }^{\circ}$ with respect to the $a$ axis. Releasing the constraints did not lead to any noticeable improvement of the fit. This structure is different from the one found previously for $\mathrm{BiCrO}_{3}$, which was also a G-type structure but with spins within the $(b, c)$ plane. ${ }^{6}$ Although the quality of the fits is similar for both magnetic structures, the latter is not compatible with group theory. Therefore we have re-examined the NPD data of Ref. 6 for $\mathrm{BiCrO}_{3}$ applying the correct symmetry constraints. For both compounds, the refined magnetic moment of $\mathrm{Cr}^{3+}$ is 2.50 (5) $\mu_{B}$. The temperature dependence of the neutron powder diffraction patterns evidences a spin reorientation transition below ca. $83 \mathrm{~K}$ for $\mathrm{Bi}_{0.99} \mathrm{Y}_{0.01} \mathrm{CrO}_{3}$ and ca. $80 \mathrm{~K}$ for $\mathrm{BiCrO}_{3}$. The refinements indicate, for both compounds, a rotation of the spin direction within the $(a, c)$ plane, the spins being aligned along the $a$ axis above $T_{\mathrm{SR}}$. The refined $\Theta$ angle between the spin direction and the $a$ axis is displayed as function of temperature on Fig. 7 for $\mathrm{Bi}_{0.99} \mathrm{Y}_{0.01} \mathrm{CrO}_{3}$. It is worth noting that the direction of the spins at $2 \mathrm{~K}$ is very close to the axis of the pseudocubic cell.

To summarize these results, all magnetic structures can be viewed as pseudocubic arrangements of magnetic $\mathrm{Cr}^{3+}$ cations 
with a G-type antiferromagnetic structure. Surprisingly, for compounds displaying the same orthorhombic Pnma symmetry, we found two different magnetic representations with different orientations of the magnetic moments: for an yttrium content lower than $x \leqslant 0.2$, spins are aligned along $b$, whereas for an yttrium content higher than $x \geqslant 0.5$, spins are aligned along $c$. To better understand the influence of changes in the nuclear structure related to the cation content on the magnetic arrangement, we have performed a symmetry adapted analysis of the octahedral distortions.

\section{Symmetry adapted mode analysis}

Many crystalline structures can be considered pseudosymmetric with respect to some configuration of higher symmetry. A group-subgroup relation exists between the space groups of the parent and the observed structures, and the distorted structure can be described as the parent crystalline structure plus a static symmetry-breaking structural distortion. The distortion relating both phases can be decomposed into contributions from different modes with symmetries given by the IRs of the parent space group. This is the starting point of the well-known Landau theory, based on the identification of the order parameter, i.e., the mode(s) driving the stabilization of the distorted phase. In general, a structure description in terms of symmetry modes separates the correlated atomic displacements which are fundamental for the phase stability from those which are marginal. It introduces a physical hierarchy among the structural parameters that can be valuable for investigating the physical mechanisms which stabilize these phases. Furthermore, the set of structural parameters used in a mode description for a distorted phase is, in general, better adapted for a controlled refinement of the structure. The separation of different symmetry modes of a distortion can be achieved by so-called symmetry-mode analysis.

The structures adopted by $\mathrm{Bi}_{1-x} \mathrm{Y}_{x} \mathrm{CrO}_{3}$ compounds are distortions of the perovskite structure and have as their parent structure the ideal cubic perovskite with space group $P m \overline{3} m$. The $\mathrm{ABX}_{3}$ perovskite structure comprises a three-dimensional network of corner-linked $\mathrm{BX}_{6}$ octahedra with A cations occupying the cavities within the network. In the ideal cubic perovskite structure the A cations are 12-coordinated, forming $\mathrm{AX}_{12}$ regular cuboctahedra. In perovskite compounds with rather rigid $\mathrm{BX}_{6}$ octahedra, the distortion modes can be fully determined by symmetry-mode analysis and can be identified with tilts or rigid unit modes of the octahedral network. This analysis provides a clear and unambiguous way to separate the effects of distortion and tilting of octahedra in

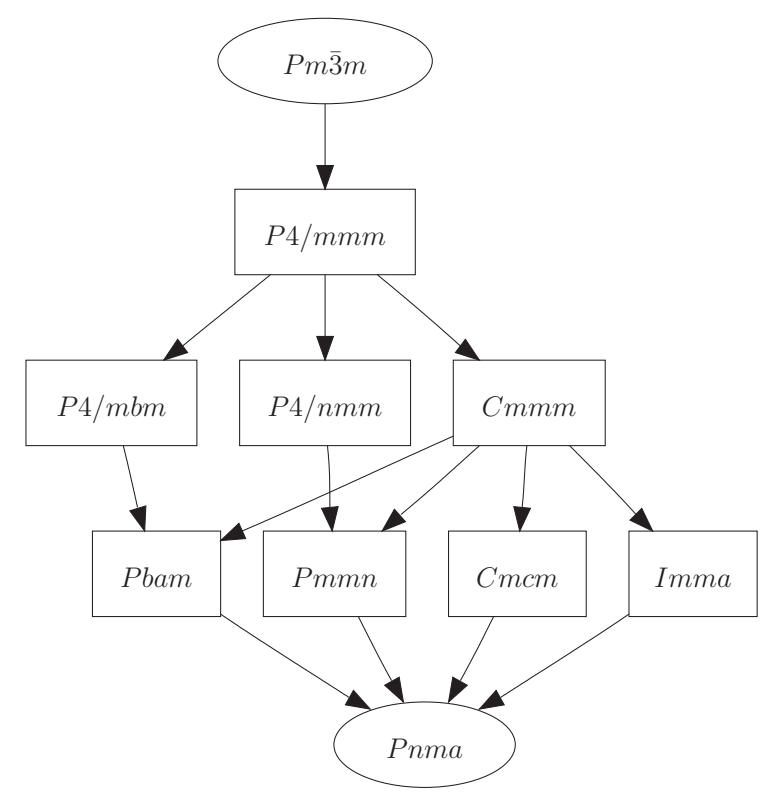

FIG. 9. Graph of maximal subgroups relating the space groups of the ideal cubic perovskite $(P m \overline{3} m)$ and the Pnma phase of $\mathrm{Bi}_{1-x} \mathrm{Y}_{x} \mathrm{CrO}_{3}$. Figure obtained with SUBGROUP-GRAPH. ${ }^{27}$

perovskites because, by definition, the modes are orthogonal to one another. The second advantage of this parametrization is that the degrees of freedom required by the perovskite structure can be rigorously derived. We performed the following symmetry-mode analysis with AMPLIMODES, ${ }^{22,23}$ a computer program available on the Bilbao Crystallographic Server [http://www.cryst.ehu.es]. ${ }^{24,25}$

\section{Samples with orthorhombic symmetry}

Let us consider first the $\mathrm{Bi}_{1-x} \mathrm{Y}_{x} \mathrm{CrO}_{3}$ compounds, which have, at room temperature, a distorted perovskite structure with Pnma symmetry. The transformation matrix (P, rotational part; $\mathbf{p}$, origin shift) relating the conventional bases of the low-symmetry (L) Pnma space group (No. 62) and the high-symmetry (H) $P m \overline{3} m$ supergroup (No. 221) defined by $(\mathbf{a}, \mathbf{b}, \mathbf{c})_{L}=(\mathbf{a}, \mathbf{b}, \mathbf{c})_{H} \mathbf{P}$ is

$$
\left(\begin{array}{ccc|c}
1 & 0 & -1 & 0 \\
0 & 2 & 0 & 0 \\
1 & 0 & 1 & 0
\end{array}\right) .
$$

The unit cell volume is multiplied by a factor of 4. In Fig. 9, the graph of maximal subgroups connecting the two space

TABLE IV. Summary of mode decomposition of the $\mathrm{YCrO}_{3}$ compound with a Pnma structure, indicating the amplitude $(\AA)$ of all intervening irreducible representation (IR) distortion components at room temperature.

\begin{tabular}{lcccc}
\hline \hline $\mathbf{K}$ vector & IR & Isotropy subgroup & Dimension & Amplitude $(\AA)$ \\
\hline$\left(\frac{1}{2}, \frac{1}{2}, \frac{1}{2}\right)$ & $\mathrm{R} 4+$ & $\operatorname{Imma}(74)$ & 1 & 1.63 \\
$\left(\frac{1}{2}, \frac{1}{2}, \frac{1}{2}\right)$ & $\mathrm{R} 5+$ & $\operatorname{Imma}(74)$ & 2 & 0.38 \\
$\left(0, \frac{1}{2}, 0\right)$ & $\mathrm{X} 5+$ & $\operatorname{Cmcm}(63)$ & 2 & 0.82 \\
$\left(\frac{1}{2}, \frac{1}{2}, 0\right)$ & $\mathrm{M} 2+$ & $P 4 / m b m(127)$ & 1 & 0.03 \\
$\left(\frac{1}{2}, \frac{1}{2}, 0\right)$ & $\mathrm{M} 3+$ & $P 4 / m b m(127)$ & 1 & 1.13 \\
\hline \hline
\end{tabular}




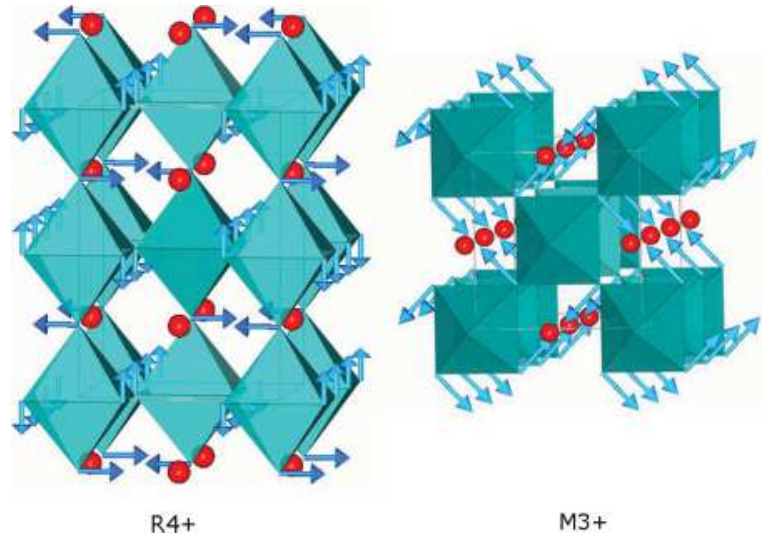

FIG. 10. (Color online) Scheme of the polarization vectors of the two primary IR distortion (R4+, M3+) components present in the Pnma structure of $\mathrm{Bi}_{1-x} \mathrm{Y}_{x} \mathrm{CrO}_{3}$.

groups is displayed. It can be seen that a single IR distortion component is not sufficient to explain the full symmetry break of the transformation from the cubic to the orthorhombic phase. That is, the Pnma phase cannot be generated by a single mode distortion, but at least two different normal modes must be active, i.e., two order parameters must be involved. This situation is similar to the very well-studied case of $\mathrm{SrZrO}_{3}$ (see Perez-Mato et al. ${ }^{23}$ and references herein).

The mode decomposition of the $\mathrm{YCrO}_{3}$ experimental $P n m a$ structure with respect to its $P m \overline{3} m$ parent structure is summarized in Table IV. Five different IRs corresponding to three different symmetry points at the border of the cubic Brillouin zone are possible. The global distortion amplitude is $2.18 \AA$ A. Considering the relative amplitude of the IRs, one can see that two distortion amplitudes are much higher: R4+ and M3+. A scheme of the distortion modes is shown in Fig. 10. The R4+ distortion corresponds to the tilt of octahedra around the $a$ direction of the orthorhombic unit cell with the Pnma space group setting $\left(a^{-} a^{-} c^{0}\right.$ in Glazer notation $\left.{ }^{26}\right)$, and M3+ corresponds to the tilt of octahedra around the $b$ direction $\left(a^{0} a^{0} c^{+}\right)$. The secondary mode X5+ and R5+ have significant nonzero amplitudes. They involve both oxygen and yttrium

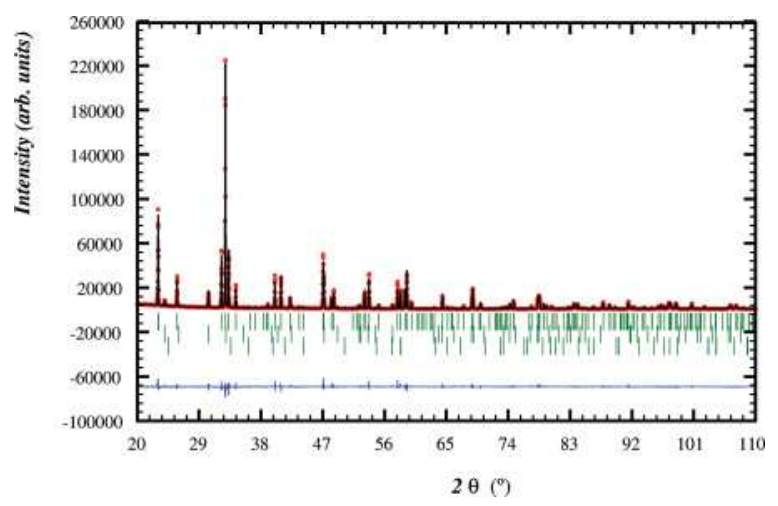

FIG. 11. (Color online) Rietveld refinement of $\mathrm{x}$-ray powder diffraction pattern of a $\mathrm{Bi}_{0.8} \mathrm{Y}_{0.2} \mathrm{CrO}_{3}$ compound adopting the Pnma structure at room temperature. Amplitudes of the basis symmetry modes generated by AMPLIMODES were directly refined in Fullprof. Bragg $R$ factor for a $\mathrm{Bi}_{0.8} \mathrm{Y}_{0.2} \mathrm{CrO}_{3}$ phase of 5.50. Weight fractions of impurities: $\mathrm{CBi}_{2} \mathrm{O}_{5}, 7.9(1) \%$; and $\mathrm{Cr}_{2} \mathrm{O}_{3}, 1.6(2) \%$.

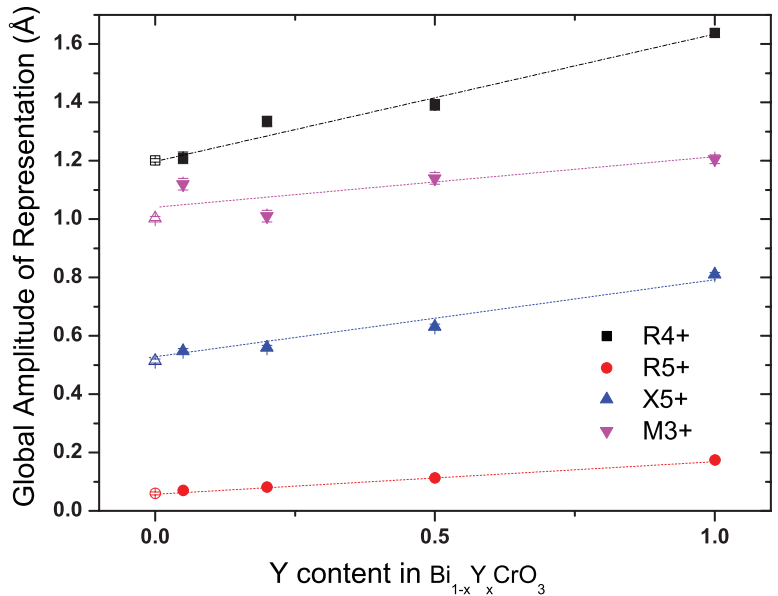

FIG. 12. (Color online) Amplitude of distortion modes of $\mathrm{Bi}_{1-x} \mathrm{Y}_{x} \mathrm{CrO}_{3}$ samples adopting a Pnma distorted perovskite structure at room temperature (filled symbols). Open symbols correspond to the amplitude of distortion modes for $\mathrm{BiCrO}_{3}(x=0)$ determined at $430 \mathrm{~K}^{6}$ in the Pnma structure. Lines are guide for the eye.

atom displacements. X5+ implies some distortion of the octahedra and alternating displacements of the $\mathrm{Y}$ atoms along the $a$ direction. R5 + mainly involves alternating displacements of the $\mathrm{Y}$ atoms along the orthorhombic $c$ direction. The amplitude of the M2+ mode is 0 within the experimental resolution. We used the implementation of AMPLIMODE in FullProf to enable a direct Rietveld refinement of the amplitudes of the basis symmetry modes generated by AMPLIMODE, instead of performing a conventional refinement of individual atomic coordinates. The amplitude of the $\mathrm{M} 2+$ mode was not refined and fixed to 0 . The refinements were carried out with the room-temperature $\mathrm{x}$-ray powder diffraction patterns of selected $\mathrm{Bi}_{1-x} \mathrm{Y}_{x} \mathrm{CrO}_{3}$ compounds. The result of a typical refinement is shown in Fig. 11. These refinements involve less parameters without degrading the quality of the fit compared to conventional analysis. Figure 12 shows the evolution of the amplitudes of the distortion modes as a function of the yttrium content $x$. For the sake of comparison we also add points obtained for $\mathrm{BiCrO}_{3}$ in its high-temperature phase (Pnma) refined from neutron powder diffraction data at $430 \mathrm{~K}$. A clear decrease in the amplitudes for all modes of distortion is observed when $x$ decreases (including the $\mathrm{BiCrO}_{3}$ sample), which seems to follow a linear trend. The decrease is less pronounced for the $\mathrm{M} 3+$ primary mode $(\approx-17 \%$ from $x=1$ to $x=0)$ than for the other primary mode, $\mathrm{R} 4+(\approx-25 \%)$. Surprisingly, the less distorted $\mathrm{BiCrO}_{3}$ compound transforms to a lower symmetry phase at lower temperature, whereas the much more distorted structure of $\mathrm{YCrO}_{3}$ persists down to low temperatures. This point is discussed later.

\section{Samples with monoclinic symmetry}

Let us now consider the mode of distortions in compounds with monoclinic symmetry at room temperature, namely, $\mathrm{BiCrO}_{3}$ and $\mathrm{Bi}_{0.99} \mathrm{Y}_{0.01} \mathrm{CrO}_{3}$. We first tried to establish the group-subgroup relationship between the high-temperature orthorhombic phase Pnma and the low-temperature monoclinic phase $C 2 / c$. No relationship between these two crystallographic space groups exists. This result implies two 


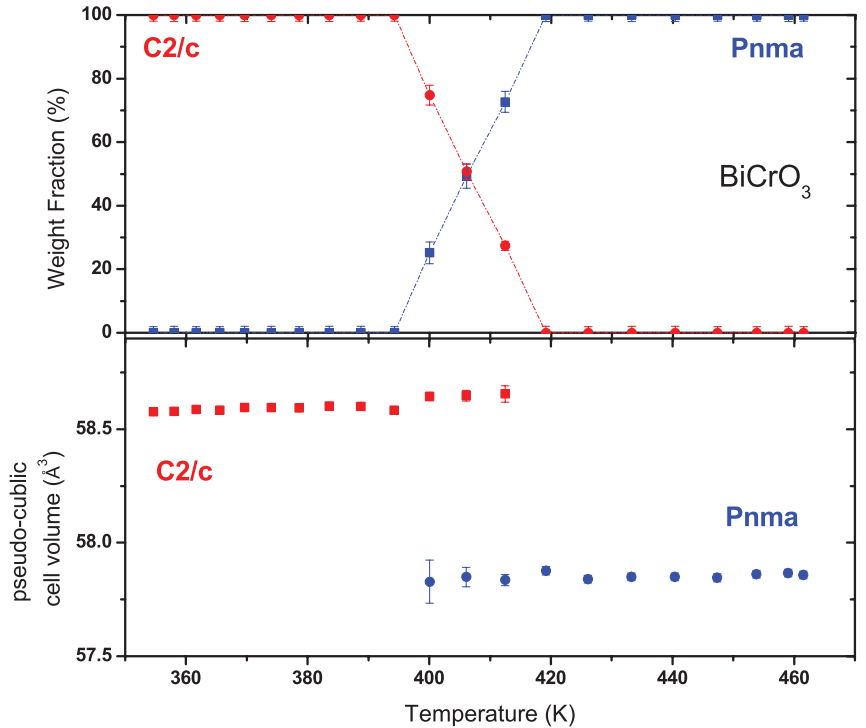

FIG. 13. (Color online) Temperature evolution of the weight fraction (top) and pseudocubic unit cell volume (bottom) of $\mathrm{BiCrO}_{3}$ across the transition between the low-temperature $C 2 / c$ and the high-temperature Pnma phases.

possibilities: (i) the determination of the space group of the low temperature monoclinic structure is wrong, or (ii) the structural transition is not a second-order transition but a first-order one. Diffraction data evidence, without any doubt, that the low-temperature structure belongs to the monoclinic system and the systematic extinctions indicate the C-centered mode. Two C-centered monoclinic space groups are subgroups of Pnma: $C 2$ (No. 9) and $C m$ (No. 8). However, there is no transformation possible between Pnma and these two groups considering the index of the transformation. In other words, no second-order transition to C-centered monoclinic structure is possible considering the observed cell. Attempts to refine the structure with the $C 2$ and $C m$ space groups do not lead to any improvements. In the following, we consider, as have previous authors, ${ }^{5,6}$ that the space group is $C 2 / c$. To examine the second hypothesis, we reinvestigate the neutron diffraction data of $\mathrm{BiCrO}_{3}$ at high temperatures published by Darie et al. ${ }^{6}$ We performed cyclic Rietveld refinement of the temperature dependence of powder neutron diffraction across the structural phase transition. The results are summarized in Fig. 13. The upper graph evidences a large range $(\approx 15) \mathrm{K}$ of phase coexistence between $C 2 / c$ and Pnma. The lower graph shows the evolution of the pseudocubic cell volume vs temperature. A clear discontinuous volume change happens at the transition. It is remarkable that the transition is associated with a negative thermal expansion. Furthermore, hysteretic behavior upon cooling and heating (not shown) was observed. All these elements demonstrate the first-order character of the structural phase transition. A symmetry-mode analysis between these two space groups is then impossible, Landau theory describing only second-order transitions.

To determine the distortion mode of the monoclinic sample, we then had to perform symmetry-mode analysis with respect to the ideal cubic perovskite $(P m \overline{3} m)$. The group-subgroup relationship between $C 2 / c$ and $P m \overline{3} m$ is rather complex as illustrated by the graph of the maximal subgroups in Fig. 14. Note that the Pnma space group does not appear on this graph. The transformation matrix $(\mathbf{P}, \mathbf{p})$ relating the conventional bases of the low-symmetry $C 2 / c$ subgroup (No. 15) and the high-symmetry $P m \overline{3} m$ supergroup (No. 221) is

$$
\left(\begin{array}{ccc|c}
1 & 1 & 1 & 1 \\
-1 & 1 & -1 & -\frac{1}{2} \\
-2 & 0 & 2 & -\frac{3}{2}
\end{array}\right)
$$

The unit cell volume is multiplied by a factor of 8 . Several IR distortion components can contribute to the symmetry break $P m \overline{3} m \longrightarrow C 2 / c$, a combination of at least two distortions belonging to different IRs is necessary. The monoclinic structure requires a basis of 13 symmetry modes, and their distribution into IR types is reported in Table V. The amplitudes of the IR distortion components present in the

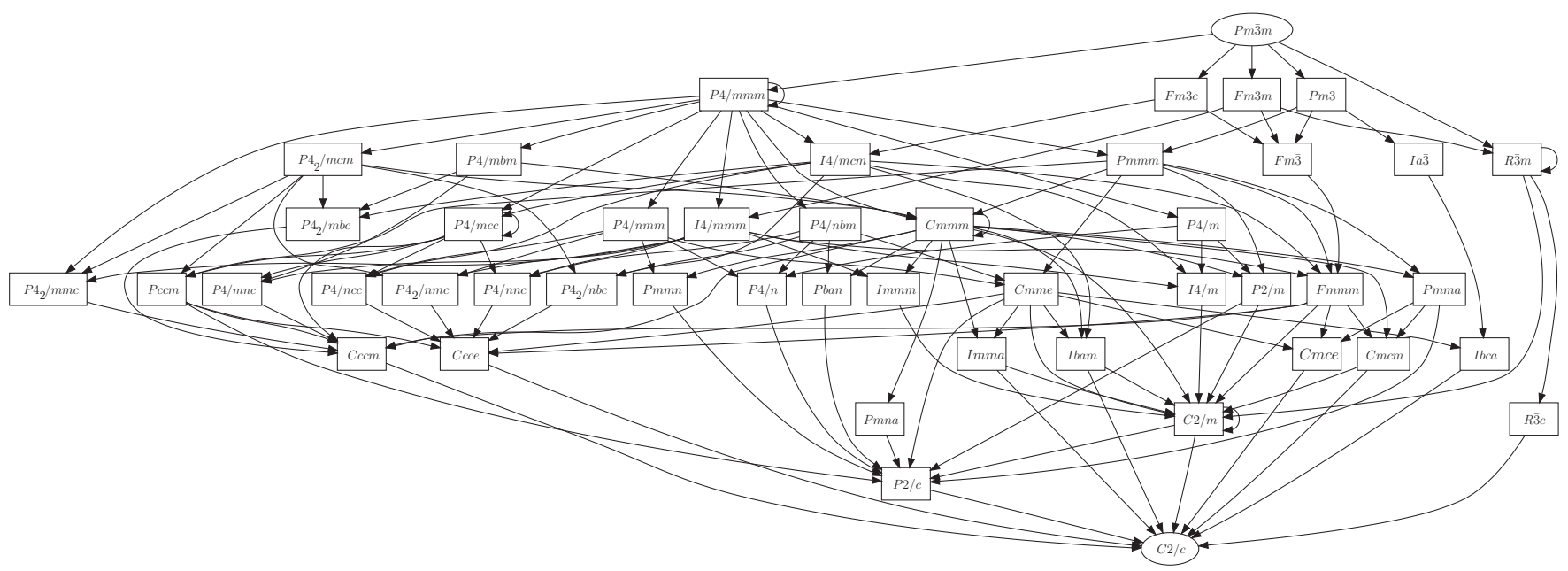

FIG. 14. Graph of maximal subgroups relating the space groups of the ideal cubic perovskite $(P m \overline{3} m)$ and the $C 2 / c$ phase of $\mathrm{Bi}_{1-x} \mathrm{Y}_{x} \mathrm{CrO}_{3}$. Note that the Pnma space group does not appear in this graph. Figure obtained with SUBGROUP-GRAPH. ${ }^{27}$ 
TABLE V. Summary of the basis modes in the distortion of $\mathrm{BiCrO}_{3}$, distributed per type of Wyckoff position (WP).

\begin{tabular}{lcc}
\hline \hline Atom $\mathrm{WP}$ & $\mathrm{Mode}(\mathrm{s})$ \\
\hline $\mathrm{O}$ & $3 d$ & $\mathrm{LD} 2(1), \mathrm{LD} 3(3), \mathrm{R} 1+(1), \mathrm{R} 3+(1), \mathrm{R} 4+(1), \mathrm{R} 5+(2)$ \\
$\mathrm{Bi}$ & $1 b$ & $\mathrm{LD} 3(1), \mathrm{R} 5+(2)$ \\
$\mathrm{Cr}$ & $1 a$ & $\mathrm{LD} 3(1)$ \\
\hline \hline
\end{tabular}

$C 2 / c$ experimental room-temperature structure of $\mathrm{BiCrO}_{3}$ calculated by AMPLIMODES are reported in Table VI. The total distortion amplitude is $1.54 \AA$. The mode decomposition evidences the hierarchy of the six intervening IR distortion components. The two primary ones can be identified as those with the highest amplitudes: R4+ and LD3. Modes R5+ and LD2 are weak and can be considered as secondary modes. $\mathrm{R} 1+$ and $\mathrm{R} 3+$ are 0 within experimental resolution. Rietveld refinement using the AMPLIMODES description in FullProf was done using D2B (ILL) neutron powder diffraction data of $\mathrm{BiCrO}_{3}$ taken at room temperature. The amplitude of $\mathrm{R} 1+$ and $\mathrm{R} 3+$ was fixed at 0 . Refined amplitudes of the representations are presented in Table VI. Their values are very close to those found via AMPLIMODE, taking into account the structure refined by Darie et al. ${ }^{6}$

The R4+ distortion mode is the same and has the same order of magnitude than the one present in the Pnma phase. It consists of collective tilting of the oxygen octahedra (see Fig. 10). The LD3 mode is a complicated mode (dimension 5) involving displacements of $\mathrm{Bi}, \mathrm{Cr}$, and a complex distortion of oxygen octahedra. LD3 is related to the isotropy subgroup $C 2 / c$, that is, $C 2 / c$ comprises the operations which leave invariant the five basis vectors in the LD3 mode. The presence of the LD3 mode alone would be sufficient to explain the full symmetry break of the transformation from the cubic to the monoclinic phase. LD3 mode is represented in Fig. 15. This distortion mode differentiates the two chromium sites: for the first one (in blue in Fig. 15; $4 d$ site) the $\mathrm{Cr}^{3+}$ cations remain centered in their oxygen coordination octahedra, which are tilted. For the second one (in green; $4 e$ site) the $\mathrm{Cr}^{3+}$ cations are off-center and their coordination octahedra are distorted. The relative displacement of the chromium ion within the oxygen octahedral cage is $0.0003 \AA$. The shift is antiparallel along the $y$ axis, the four local electric dipoles canceling each other. The displacement of $\mathrm{Bi}$ within the oxygen dodecahedra is much more important and complex. The distance between $\mathrm{Bi}^{3+}$ and the barycenter of the 12 surrounding $\mathrm{O}^{2-}$ anions is $0.4049 \AA$. The eight local electric dipoles are oriented in
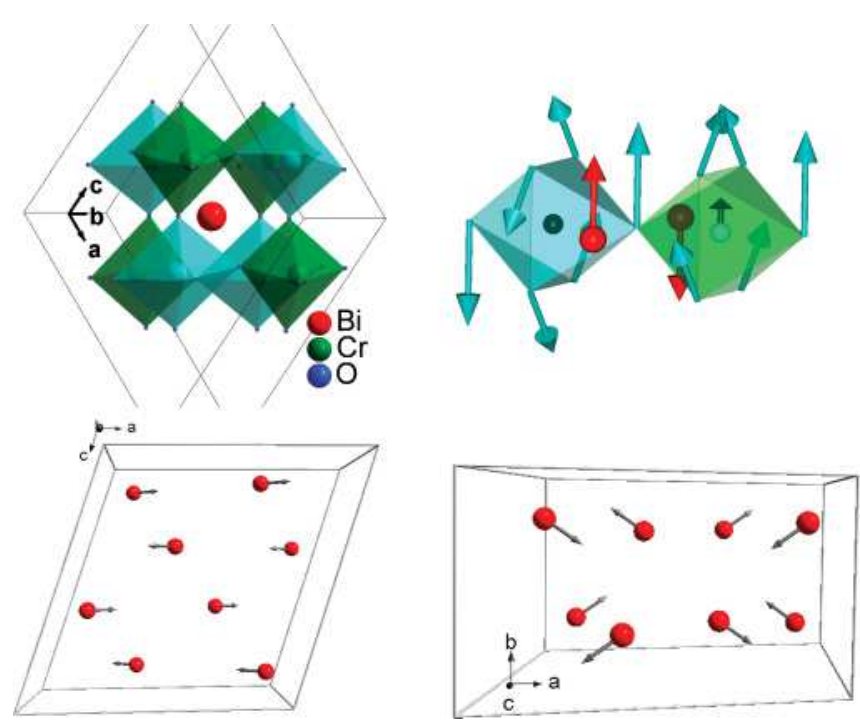

FIG. 15. (Color online) Top left: LD3 distortion mode represented in the pseudocubic unit of a monoclinic cell. Top right: Detailed view of the LD3 distortion around the two distinct chromium sites. The directions of atomic displacement correspond to the direction of the arrows. The figure was prepared using the program FPstudio included in the FullProf suite. Bottom: Scheme of an LD3 antiferroelectric arrangement. $\mathrm{Bi}$ atoms are (red) spheres; arrows represent local electric dipoles due to the relative displacement of the bismuth ion within the oxygen dodecahedral cage.

opposite directions in the $a b$ plane (see arrows in the bottom panels in Fig. 15) and the total dipole moment is 0 within the cell, as follows from the inversion center that is present at the $4 d \mathrm{Cr}^{3+}$ ionic site. The LD3 mode is therefore responsible for the antiferroelectric arrangement in monoclinic $\mathrm{BiCrO}_{3}$.

\section{DISCUSSION}

The phase diagram reported in Fig. 16 summarizes the main results obtained for the magnetic and structural properties of the $\mathrm{Bi}_{1-x} \mathrm{Y}_{x} \mathrm{CrO}_{3}$ solid solution. Let us consider first the effect of yttrium substitution on the crystal structure of $\mathrm{BiCrO}_{3}$. We found that less than $5 \%$ of yttrium is sufficient to suppress the monoclinic structure and stabilize the high-temperature orthorhombic structure down to $2 \mathrm{~K}$. This is an indication that $\mathrm{BiCrO}_{3}$ is on the verge of a structural instability. This instability is evidenced also in the results of Belik et al., ${ }^{28}$ who found in their polycrystalline $\mathrm{BiCrO}_{3}$ ceramic $10 \%$ to $15 \%$ of the orthorhombic phase in addition to the monoclinic one. Surpris-

TABLE VI. Summary of the mode decomposition of a $\mathrm{BiCrO}_{3}$ compound with $C 2 / c$ structure, indicating the amplitude $(\AA)$ of all intervening irreducible representation (IR) distortion components at room temperature.

\begin{tabular}{lcccc}
\hline \hline K vector & IR & Isotropy subgroup & Amplitude $(\AA)$ & Refined amplitude $(\AA)$ \\
\hline$\left(\frac{1}{2}, \frac{1}{2}, \frac{1}{2}\right)$ & LD2 & $R-3 c(167)$ & 0.12 & $0.10(2)$ \\
$\left(\frac{1}{2}, \frac{1}{2}, \frac{1}{2}\right)$ & LD3 & $C 2 / c(15)$ & 0.82 & $0.79(1)$ \\
$\left(\frac{1}{2}, \frac{1}{2}, \frac{1}{2}\right)$ & $\mathrm{R} 1+$ & $F m-3 m(225)$ & 0.01 & 0.00 \\
$\left(\frac{1}{2}, \frac{1}{2}, \frac{1}{2}\right)$ & $\mathrm{R} 3+$ & $I 4 / m m m(139)$ & 0.02 & 0.00 \\
$\left(\frac{1}{2}, \frac{1}{2}, \frac{1}{2}\right)$ & $\mathrm{R} 4+$ & $\mathrm{Imma}(74)$ & 1.28 & $1.31(1)$ \\
$\left(\frac{1}{2}, \frac{1}{2}, \frac{1}{2}\right)$ & $\mathrm{R} 5+$ & $\mathrm{C} 2 / \mathrm{m}(74)$ & 0.20 & $0.19(1)$ \\
\hline \hline
\end{tabular}




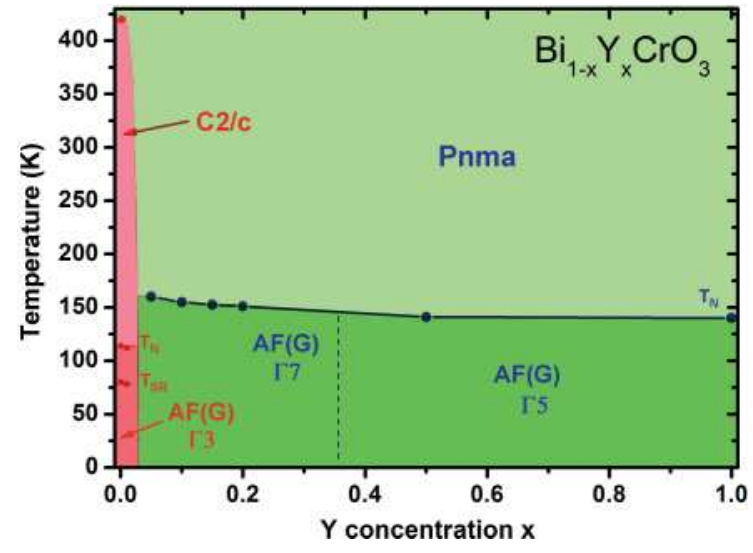

FIG. 16. (Color online) Phase diagram of $\mathrm{Bi}_{1-x} \mathrm{Y}_{x} \mathrm{CrO}_{3}$. AF: antiferromagnetic states. $T_{N}$ and $T_{\mathrm{SR}}$ denote the respective Néel and spin reorientation transition temperatures.

ingly, the total amplitude of the distortion for the orthorhombic phase compared to the parent ideal cubic structure is lower in $\mathrm{BiCrO}_{3}$ than in $\mathrm{YCrO}_{3}$, although only $\mathrm{BiCrO}_{3}$ undergoes a structural phase transition which lowers its symmetry. This symmetry break may be associated with the lone-pair effect of bismuth. The stereoactive $\mathrm{Bi} 6 s^{2}$ lone pair probably affects the stability of the orthorhombic phase by creating additional distortion modes such as LD3 with a monoclinic isotropy subgroup, leading to the symmetry-lowering transition. The yttrium substitution leads progressively to a dilution of the collective lone-pair effect and suppresses this instability. The particular monoclinic distorted perovskite structure of $\mathrm{BiCrO}_{3}$ is found only in two other Bi-based perovskites: $\mathrm{BiMnO}_{3}$ and $\mathrm{BiScO}_{3} .{ }^{29,30} \mathrm{BiFeO}_{3},{ }^{31} \mathrm{BiCoO}_{3},{ }^{29}$ and $\mathrm{BiNiO}_{3}{ }^{32}$ present different distortions. However, all these compounds possess the Pnma symmetry phase as the parent structure at either high temperature or high pressure. One can suggest that the local distortion at the Bi site brought about by the presence of the electronic lone pair does not couple the same way to the lattice depending on the nature of the octahedrally coordinated cations, leading to different low-temperature crystal structures.

The magnetic properties of $\mathrm{Bi}_{1-x} \mathrm{Y}_{x} \mathrm{CrO}_{3}$ are also related to structural distortions. The Néel temperature in the solid solution domain with Pnma symmetry decreases from $160 \mathrm{~K}$ for $\mathrm{Bi}_{0.95} \mathrm{Y}_{0.05} \mathrm{CrO}_{3}$ to $140 \mathrm{~K}$ for $\mathrm{YCrO}_{3}$. The super exchange interactions between $\mathrm{Cr}^{3+}$ magnetic cations mediated by oxygen anions control the ordering temperature. The strength of these interactions is expected to depend strongly on the $\mathrm{Cr}-\mathrm{O}-\mathrm{Cr}$ bond angles. The primary distortion modes revealed by symmetry adapted analysis of the Pnma structure, R4+ and $\mathrm{M} 3+$, represent tilts of the $\mathrm{CrO}_{6}$ octahedra. The observed increase in amplitudes for the R4+ and M3+ distortion modes with increasing yttrium content (see Fig. 12) corresponds to the $\mathrm{Cr}-\mathrm{O}-\mathrm{Cr}$ bond angles progressively shifting away from their ideal $180^{\circ}$ value. The two different $\mathrm{Cr}-\mathrm{O}-\mathrm{Cr}$ angles have approximately the same values and vary from ca. $153^{\circ}$ for $x=0$ to ca. $145^{\circ}$ for $x=1$. There is therefore a direct correlation between the increase in the amplitude of distortion and the decrease in the magnetic ordering temperature. The spin arrangements adopted throughout the $\mathrm{Bi}_{1-x} \mathrm{Y}_{x} \mathrm{CrO}_{3}$ solid solution are all G-type antiferromagnets, however, the direction of the spins changes with the yttrium content: spins are aligned along the $b$ axis ( $\Gamma_{7}$ magnetic representation) for yttrium-poor compounds and along the $c$ axis $\left(\Gamma_{5}\right.$ magnetic representation) for yttrium-rich compounds. The origin of the anisotropy of $\mathrm{Cr}^{3+}$ spins can be attributed to single-ion anisotropy. This magnetocrystalline anisotropy is determined by the interaction between the orbital state of a magnetic $\mathrm{Cr}^{3+}$ cation and the surrounding crystalline field created by its oxygen octahedron. Any distortion of the oxygen octahedra can then affect the anisotropy of $\mathrm{Cr}^{3+}$ cations and therefore the direction of the spins. In Pnma symmetry, the only active mode which distorts the octahedra is X5+. In this mode, apical oxygen anions are displaced alternately along the $a$ direction while basal oxygen anions do not move. The amplitude of the X5+ mode increases with yttrium substitution and may be responsible for the observed change in the spin direction. For all samples a weak ferromagnetic behavior is observed in magnetization measurements and therefore spins must be canted in all G-type structures. This effect was not observed in our neutron powder diffraction experiments due to the weakness of the resulting moments involved. The small deviation from collinearity could be explained by invoking Dzyaloshinskii-Moriya interactions, which are allowed for all the $\mathrm{Bi}_{1-x} \mathrm{Y}_{x} \mathrm{CrO}_{3}$ structures.

\section{CONCLUSION}

In this work, the effect of the substitution of yttrium for bismuth in the $\mathrm{BiCrO}_{3}$ distorted perovskite compound was investigated. The room-temperature monoclinic structure of $\mathrm{BiCrO}_{3}$ (space group $C 2 / c$ ) is suppressed for an yttrium content of 5\%. For larger substitutions, the high-temperature orthorhombic structure (space group Pnma) is stabilized down to $2 \mathrm{~K}$. Magnetization measurements show that the samples order antiferromagnetically with a weak ferromagnetic behavior. Neutron powder diffraction experiments revealed that all compounds display G-type antiferromagnetic structures. For the compounds with orthorhombic symmetry, two different magnetic representations with different spin directions were found, depending on the yttrium content. $\mathrm{BiCrO}_{3}$ and $\mathrm{Bi}_{0.99} \mathrm{Y}_{0.01} \mathrm{CrO}_{3}$ have the same magnetic representation and present a similar spin reorientation transition within the $(a, c)$ plane. Symmetry-adapted analysis was used to investigate the main distortion modes from the ideal cubic perovskite structure. The amplitudes of distortion components increase significantly with yttrium substitution and were related to the decrease in Néel temperature. For a low yttrium content, the $C 2 / c$ monoclinic space group presents no groupsubgroup relationship with the Pnma space group of the high-temperature phase. Close examination of the structural transition reveals a first-order character. The symmetry adapted analysis of the $C 2 / c$ distortions from the ideal cubic structure reveals the importance of the LD3 mode with a monoclinic isotropy subgroup, which is responsible for antiferroelectricity and might be related to the effect of the stereoactive $\mathrm{Bi} 6 s^{2}$ electronic lone pair. The suppression of this distortion mode (and thus of the monoclinic phase) with increasing yttrium content would be related to the dilution of the lone-pair effect and its coupling to the lattice. 


\section{ACKNOWLEDGMENTS}

We would like to gratefully acknowledge scientific and technical support we received during the neutron powder diffraction experiments from V. Nassif and L. Laversenne at CRG D1B (ILL) and G. André at G 4-1 (LLB). C.V.C. thanks G. Nénert for useful discussions on AMPLIMODE.
*Corresponding author: claire.colin@grenoble.cnrs.fr

${ }^{1}$ F. Sugawara, S. Iida, Y. Syono, and S.-i. Akimoto, J. Phys. Soc. Jpn. 20, 1529 (1965).

${ }^{2}$ F. Sugawara, S. Iiida, Y. Syono, and S.-i. Akimoto, J. Phys. Soc. Jpn. 25, 1553 (1968) .

${ }^{3}$ N. A. Hill, P. Bättig, and C. Daul, J. Phys. Chem. B 106, 3383 (2002).

${ }^{4}$ S. Niitaka, M. Azuma, M. Takano, E. Nishibori, M. Takata, and M. Sakata, Solid State Ionics 172, 557 (2004).

${ }^{5}$ A. Belik, S. Iikubo, K. Kodama, and N. Igawa, Chem. Mater. 20, 3765 (2008).

${ }^{6}$ C. Darie, C. Goujon, M. Bacia, H. Klein, P. Toulemonde, P. Bordet, and E. Suard, Solid State Sci. 12, 660 (2010).

${ }^{7}$ Y. Xu, X. Hao, J. Meng, and D. Zhou, J. Phys.: Condens. Matter 21, 236006 (2009).

${ }^{8}$ V. M. Judin and A. B. Sherman, Solid State Commun. 4, 661 (1966).

${ }^{9}$ E. F. Bertaut, G. Bassi, G. Buisson, P. Burlet, and J. Chappert, J. Appl. Phys. 37, 1038 (1966).

${ }^{10}$ K. Ramesha, A. Llobet, T. Proffen, C. R. Serrao, and C. N. R. Rao, J. Phys.: Condens. Matter 19, 102202 (2007).

${ }^{11}$ C. R. Serrao, A. K. Kundu, S. B. Krupanidhi, U. V. Waghmare, and C. N. R. Rao, Phys. Rev. B 72, 220101 (2005).

${ }^{12}$ A. Durán, A. Arévalo-López, E. Castillo-Martínez, M. GarcíaGuaderrama, E. Moran, M. Cruz, F. Fernández, and M. AlarioFranco, J. Solid State Chem. 183, 1863 (2010).

${ }^{13}$ C. Goujon, C. Darie, M. Bacia, H. Klein, L. Ortega, and P. Bordet, J. Phys.: Conf. Ser. 121, 022009 (2008).

${ }^{14}$ V. Bedekar, R. Shukla, and A. K. Tyagi, Nanotechnology 18, 155706 (2007).

${ }^{15}$ J. Rodríguez-Carvajal, Physica B 192, 55 (1993).

${ }^{16}$ E. F. Bertaut, Acta Crystallogr. Sec. A 24, 217 (1968).
${ }^{17}$ A. S. Wills, Physica B 278, 680 (2000).

${ }^{18}$ J. Rodríguez-Carvajal, BasIreps: A Program for Calculating Irreducible Representations of Space Groups and Basis Functions for Axial and Polar Vector Properties.

${ }^{19}$ E. Wollan and W. Koehler, Phys. Rev. 100, 545 (1955).

${ }^{20} \mathrm{O}$. V. Kovalev, Representations of the Crystallographic Space Groups (Gordon and Breach, New York, 1993).

${ }^{21}$ P. Brown, J. Forsyth, and F. Tasset, Solid State Sci. 7, 682 (2005).

${ }^{22}$ D. Orobengoa, C. Capillas, M. I. Aroyo, and J. M. Perez-Mato, J. Appl. Crystallogr. 42, 820 (2009).

${ }^{23}$ J. M. Perez-Mato, D. Orobengoa, and M. I. Aroyo, Acta Cryst. A 66, 558 (2010).

${ }^{24}$ M. I. Aroyo, J. M. Perez-Mato, C. Capillas, E. Kroumova, S. Ivantchev, G. Madariaga, A. Kirov, and H. Wondratschek, Z. Kristallogr. 221, 15 (2006).

${ }^{25}$ M. I. Aroyo, A. Kirov, C. Capillas, J. M. Perez-Mato, and H. Wondratschek, Acta Crystallogr. Sec. A 62, 115 (2006).

${ }^{26}$ A. M. Glazer, Acta Crystallogr. Sec. B 28, 3384 (1972).

${ }^{27}$ S. Ivantchev, E. Kroumova, G. Madariaga, J. M. Pérez-Mato, and M. I. Aroyo, J. Appl. Crystallogr. 33, 1190 (2000).

${ }^{28}$ A. A. Belik and E. Takayama-Muromachi, J. Phys.: Conf. Ser. 165, 012035 (2009).

${ }^{29}$ A. Belik, S. Iikubo, K. Kodama, N. Igawa, S.-i. Shamoto, S. Niitaka, M. Azuma, Y. Shimakawa, M. Takano, F. Izumi, and Others, Chem. Mater. 18, 798 (2006).

${ }^{30}$ A. Belik and K. Kato, J. Solid State 182, 685 (2009).

${ }^{31}$ C. Michel, J.-M. Moreau, G. D. Achenbach, R. Gerson, and W. J. James, Solid State Commun. 7, 701 (1969).

${ }^{32}$ M. Azuma, S. Carlsson, J. Rodgers, M. G. Tucker, M. Tsujimoto, S. Ishiwata, S. Isoda, Y. Shimakawa, M. Takano, and J. P. Attfield, J. Am. Chem. Soc. 129, 14433 (2007). 